\title{
New Protective Groups in Alicyclic Methacrylate Polymers for 193-nm Resists
}

\author{
Koji Nozaki and Ei Yano \\ Fujitsu Laboratories Ltd. \\ 10-1 Morinosato-Wakamiya, Atugi 243-01, Japan \\ E-mail:knozaki@flab.fujitsu.co.jp
}

\begin{abstract}
We used a methacrylate polymer with two acid labile protective groups, mevalonic lactone and 2-methyl-2-adamantanol (Fig. 1), for a 193-nm resist.[1-4] The resist demonstrated high sensitivity and a high resolution as a single layer resist. In this paper, we investigated polar five protective groups in place of the lactone group to obtain necessary properties for good lithographic performance. We discuss the impact of the polarity and acid cleavability of the protective groups to make a good match to 2-methyl-2-adamantyl group. Among the selected protective groups, we found that 3-hydroxy- $\gamma$-butyrolactone yielded acceptable lithographic performance.
\end{abstract}

Keywords: 193-nm resist, protective group, lactone, alicyclic methacrylate

\section{Introduction}

Recent advances in chemically amplified single layer resists for 193-nm lithography have been outstanding. In spite of the limitation in the matrix polymers for such short-wavelength light, transparent and etch-resistant materials have been reported in a few brief years by workers at Fujitsu [5-9], Matsushita [10], IBM/MIT team [11], NEC [12], and Toshiba [13]. Much efforts have been focused on methacrylate polymers with alicyclic pendant groups to demonstrate sub-0.2- $\mu \mathrm{m}$ resolution to date.

More recently, a new class of cycloaliphatic or cycloolefin polymers (i.e. polynorbornen) were reported for 193-nm resists by IBM-MIT-University of Texas collaboration [14], Bell Laboratories researchers [15], and workers at Samsung [16]. These polymers contain large quantities of etch-resistant alicyclic moieties directly on the polymer backbone. Therefore, it imparts a high etch resistance better than that of novolak resin, even in an aggressive chlorine plasma. Such classes of polymers are attractive from the etch-resistant perspective as alternate platforms for 193-nm resists.

Generally, 193-nm single layer resists require: (1) high optical transparency at $193 \mathrm{~nm},(2)$ good dry-etch resistance comparable to Novolak resist, and (3) thermal stability to withstand the temperature and conditions used in device manufacturing. In addi- tion, alkaline developability in a $2.38 \%$ TMAH developer is strongly required for the development process compatibility which has been established for Novolak and PVP resists.

We reported a single-layer resist material for 193$\mathrm{nm}$ lithography to meet the above-mentioned requirements.[1-4] The resist is comprised of a poly (MLMA-co-MAdMA) (Fig. 1), which contained mevalonic lactone and 2-methyl-2-adamantanol as protective groups for carboxylic acid, and $2 \mathrm{wt} \%$ of triphenylsulfonium triflate as a photoacid generator<smiles>CCC(C)(C)C(=O)OC1(C)CCOC(=O)C1</smiles>

Figure 1. Structural formula of poly(MLMA-coMAdMA)

(PAG). Using an ArF exposure system (NA=0.55), we could delineate $0.15-\mu \mathrm{m} \mathrm{L} / \mathrm{S}$ patterns at a dose of $4.8 \mathrm{~mJ} / \mathrm{cm}^{2}$ after $2.38 \%$ TMAH development with this resist. In this paper, we investigated the necessary properties of a protective group neede to make a good combination with the adamantyl groups which 
also gives good resist adhesion and lithographic performance. We selected five polar and cyclic protective groups in combination with the adamantyl group. We discussed the impact of the protective groups with or without acid cleavability for lithographic performance.

\section{Experimental}

2.1 Materials

Glycidyl methacrylate, 3-methyl- $\gamma$-butyrolactone-3-yl methacrylate, 2-hydroxy- $\gamma$-butyrolactone, 3-hydroxy- $\gamma$-butyrolactone, and 3-hydroxy tetrahydrofuran were used as received. 2-methyl-2-adamantyl methacrylate (MAdMA) was prepared using previously published methods.[3] Methacryloyl chloride was distilled under reduced pressure. Methylene chloride and triethylamine were distilled over $\mathrm{CaH}_{2}$. AIBN was purified by recrystallization in methanol. Triphenylsulfonium triflate (TPSOTf), PGMEA (propyleneglycol-1-methylether-2-acetate) and $\gamma$-butyrolactone were used as received.

\subsection{Synthesis}

The methacrylate monomers were synthesized in one-step esterification using methacryloyl chloride and corresponding alcohols in the presence of triethylamine. For example, $\gamma$-butyrolactone-2-yl methacrylate (GBLMA) was synthesized as follows: to a well dried $200-\mathrm{mL}$, four-necked flask fitted with a dropping funnel, a nitrogen gas inlet, a rubber septum, a drying tube filled with $\mathrm{CaCl}_{2}$ and a magnetic stir bar was added $5.0 \mathrm{~g}(48.9 \mathrm{mmol})$ of 2hydroxy- $\gamma$-butyrolactone, $5.45 \mathrm{~g}(53.9 \mathrm{mmol})$ of triethylamine, $50 \mathrm{~mL}$ of methylene chloride under a dry nitrogen atmosphere. The mixture was stirred at $0^{\circ} \mathrm{C}$. To this mixture, $5.11 \mathrm{~g}(48.9 \mathrm{mmol})$ of methacryloyl chloride was added dropwise through the dropping funnel for 1 hour and stirred vigorously. Then the reaction mixture was allowed to warm to room temperature and was stirred for 2 hours. The resultant mixture was washed with $100 \mathrm{~mL}$ of water. The organic layer was separated and the water layer was extracted with methylene chloride three times. The organic layer and the extracts were combined, washed with brine, dried with anhydrous $\mathrm{Na}_{2} \mathrm{SO}_{4}$, and concentrated in vacuo at $42^{\circ} \mathrm{C}$. Purification of the residue by silica gel chromatography gave $7.25 \mathrm{~g}$ $(87.1 \%)$ of GBLMA as a colorless oil. According to the literature, PCMA (2, 3-propylenecarbonate-1yl methacrylate) was synthesized in polymer reaction using poly(glycidyl methacrylate-co-2-methyl-2adamantyl methacrylate) and $\mathrm{CO}_{2}$ gas.[12]

All polymers were synthesized by free radical solution polymerization. For example, poly (GBLMA-co-MAdMA) was prepared as follows: a solu- tion of $3 \mathrm{~g}$ (17.6 mmol) of GBLMA, $3.51 \mathrm{~g}$ (14.4 mmol) of MAdMA, $10.7 \mathrm{~mL}$ of dioxane and magnetic stir bar was stirred. To this mixture was added $788 \mathrm{mg}(4.8 \mathrm{mmol})$ of AIBN and stirred at $70^{\circ} \mathrm{C}$. After 8 hours, the mixture was precipitated in $1 \mathrm{~L}$ of methanol, filtered off with a glass filter, and dried in vacuo at $45^{\circ} \mathrm{C}$ for 6 hours. The resultant white powder was dissolved in THF and then reprecipitated in $1 \mathrm{~L}$ of methanol, filtered off using a glass filter, and dried again. This reprecipitation procedure was repeated one more time to provide $5.35 \mathrm{~g}(82 \%)$ of the copolymer as a white powder.

\subsection{Analytical measurements}

NMR spectra were obtained using a JEOL GX500 spectrometer at a resonnance frequency of $500 \mathrm{MHz}$ for protons. IR spectra were obtained using a JEOL JIR-100 spectrometer. DSC and TGA were measured using a DuPont 9900 thermal analyzer with a $10^{\circ} \mathrm{C} / \mathrm{min}$ heating rate. Molecular weight determination was performed using a TOSOH SC8020 chromatogarph. UV absorption were recorded on a Hitachi U-3200 spectrophotometer with resist films spincoated on a quartz substrates.

\subsection{Resist formulation and lithographic evaluation}

Each resist consists of the copolymer, $2 \mathrm{wt} \%$ of TPSOTf, and PGMEA or ethyl lactate. The wellmixed resist solution was then filtered through a series of 0.5 - and $0.2-\mu \mathrm{m}$ Teflon membrane filters, spun onto HMDS-primed Si substrates, and baked for 60 seconds on a hot plate to form $0.5-\mu$ m-thick films. Polyolefinic resin was used for top-coats over the resist films to prevent the uptake of airborne contamination. The resist coated substrates were exposed with an ArF excimer laser exposure system (Nikon, NA $=0.55$ ), followed by a postexposure bake (PEB) for 60 seconds on a hot plate. Then the topcoats were removed in a xylene rinse. Finally, the films were developed in a $2.38 \%$ TMAH aqueous solution and rinsed in deionized water.

\section{Results and Discussions}

\subsection{Synthesis}

Yields and formulae of all monomers used in this study are listed in Table 1. Several of the monomers synthesis have not yet been optimized and as aresult the yield is lower than expected.

Table 2 shows the structural formulae, compositions, molecular weight and polydispersion of the polymers prepared in this study. We prepared each copolymer using MAdMA monomer and the synthesized monomers using the same conditions (e.g. monomer concentration, solvent and temperature). We obtained a good yield for each polymer synthe- 
Table 1. Structural Formulae and Yields of Monomers

\begin{tabular}{|c|c|c|c|c|c|}
\hline${ }_{2} \mathrm{C}=\mathrm{C}_{\prod_{\mathrm{O}}^{\prime}}^{\mathrm{CH}_{3}}$ & $R=$ & & & & \\
\hline abbreviation & GBLMA & HGBMA & 3-THFMA & PCMA* & MBLMA \\
\hline yield (\%) & 87 & 55 & 56 & - & purchased \\
\hline
\end{tabular}

* Synthesized from glycidyl methacrylate containing copolymer

Table 2. Structural Formulae and Characteristics of Polymers

\begin{tabular}{cccccc}
\hline & & & \\
& $\mathrm{GBB}$ & $3-\mathrm{THF}$ & $\mathrm{PC}$ & $\mathrm{MBL}$ \\
abbreviation & $55: 45$ & $\leftarrow$ & $\leftarrow$ & $\leftarrow$ & $\leftarrow$ \\
feed (m: $: \mathrm{n})$ & $51: 49$ & $46: 54$ & $51: 49$ & $53: 47$ & $48: 52$ \\
$\mathrm{Mw}$ & 14,900 & 29,300 & 21,300 & 14,100 & 13,600 \\
$\mathrm{Mw} / \mathrm{Mn}$ & 1.83 & 2.56 & 2.49 & 1.82 & 1.68 \\
yield (\%) & 82 & 86 & 54 & 74 & 66
\end{tabular}

sis except in 3-THF.

\subsection{Deprotection of protective groups}

We investigated a model study of the acidic deprotection of the protective groups using ${ }^{1} \mathrm{H}-\mathrm{NMR}$ spectroscopy. At first, we attempted an anhydrous condition like resist films using toluene- $d_{8}$ with camphor sulfonic acid to avoid acidic hydrolysis of the protective groups. But this condition did not work well as expected. Comparing trifluoromethanesulfonic acid (triflic acid) which was generated from the PAG in the resists, the sulfonic acid was not so strong as to start the acid deprotection. Therefore, we used deuterium chloride ( $\mathrm{DCl}, 20 \mathrm{wt} \%$ solution in $\mathrm{D}_{2} \mathrm{O}$ ) as an active acid catalyst. We prepared a solution with each monomer by dissolving in $0.7 \mathrm{~mL}$ of DMSO- $d_{6}$ with about $50 \mu \mathrm{L}$ of the $\mathrm{DCl}$ solution. In the case of PCMA, we used its homopolymer because it was synthesized by a polymer reaction.

We obtained 'H-NMR spectra with these sample as a reference. Then each sample was heated to 120 ${ }^{\circ} \mathrm{C}$ for $300 \mathrm{~s}$. We then obtained 'H-NMR spectra with the samples. We compared the difference occurred in the ${ }^{1} \mathrm{H}-\mathrm{NMR}$ spectra before and after heating of each sample. Since the peak for a vinyl proton $\left(\mathrm{H}_{2} \mathrm{C}=\mathrm{C}\right.$, approximately $\delta=6 \mathrm{ppm}$ ) of the methacrylate monomers in each monomer was observed as a separated peak, we focused on this peak in the spectra. Addi- tionally, it was suitable because the resonance frequencies were somewhat different between the esters and the resultant methacrylic acid. We compared the integral ratio of the vinyl protons and calculated the conversion to methacrylic acid.

Table 3 shows the result of the acid deprotection of the monomers. Generally, we observed the peaks for the vinyl protons of methacrylic acid at about $\delta=5.9$ and $5.6 \mathrm{ppm}$ (depends on the acidity of a solution) in the NMR spectrum. Among the protective groups, PC did not show any difference in the NMR spectrum. It seems to be stable in this condition. GBL displayed apparently different peaks $(\delta=6.1,5.7 \mathrm{ppm})$ from the monomer and methacrylic

Table 3. Acidic Deprotection of the Monomers

\begin{tabular}{cc}
\hline monomer & acid conversion (\%) \\
\hline GBL & no reaction ${ }^{* 1}$ \\
HGB & 7 \\
$3-\mathrm{THF}$ & trace \\
PC $^{* 2}$ & no reaction \\
MBL & 8.3 \\
\hline MLMA & 82.2 \\
MAdMA & 28.6
\end{tabular}

*1 An unidentified product was observed

*2 PCMA homopolymer was used 
acid. The peaks seemed to be derived from an unidentified product. The calculated conversion for this product was $27.2 \%$. Acid hydrolysis might occur at the lactone ring instead of deprotection. HGB and MBL exhibited relatively low acid conversion. 3THF showed very low acid conversion.

We also analyzed the acid conversion in MLMA and MAdMA monomers in the same manner. MLMA gave extremely high acid conversion. MAdMA also afforded sufficient acid conversion. The good reactivity of both monomers in the acidic media well explained that the excellent lithographic performance of the poly(MLMA-co-MAdMA) based resist. Although the results imply acidic hydrolysis of the protective groups, we think that they explain the acidic reactivity of the protective groups to some extent.

\subsection{Thermal stability of polymers}

Table 4 shows the thermal stabilities of the polymers. We observed a weak $T g$ (glass transition temperature) in $\mathrm{PC}$ at approximately $109^{\circ} \mathrm{C}$, while there were no $\mathrm{Tgs}$ in the others up to $200^{\circ} \mathrm{C}$ in the second heat operations. We also examined $T 5 \%(5 \%$ weight loss temperature). It is interesting that all polymers exhibit thermal stabilities as high as MAdMA homopolymer. Comparing $T 5 \%$ in poly (MLMA-coMAdMA), these polymers showed over $30^{\circ} \mathrm{C}$ higher temperatures. We attribute the difference in thermal stabilities between the five-membered ring and six-

Table 4. Thermal Characteristies of the Polymers

\begin{tabular}{ccc}
\hline polymer & $T g\left({ }^{\circ} \mathrm{C}\right)$ & $T 5 \%\left({ }^{\circ} \mathrm{C}\right)$ \\
\hline GBL & - & 217 \\
HGB & - & 206 \\
$3-$ THF & - & 215 \\
PC & 109 & 210 \\
MBL & - & 211 \\
MLMA $_{31}$-MAdMA & 105 & 176 \\
MAdMA $_{49}$ & - & 218
\end{tabular}

Heating rate: $10^{\circ} \mathrm{C} / \mathrm{min}$.

Table 5. Optical Density of the Resists

\begin{tabular}{ccc}
\hline resist $^{*}$ & O.D. $\left(\mu \mathrm{m}^{-1}\right)$ & Transmittance (\%) \\
\hline GBL & 0.677 & 21 \\
HGB & 0.615 & 24.3 \\
3-THF & 0.711 & 19.5 \\
PC & 0.572 & 26.8 \\
MBL & 0.535 & 29.2 \\
MLMA-MAdMA & 0.624 & 23.8 \\
* According to the ploymer name &
\end{tabular}

membered ring structures to the fact that the deprotection of the six-membered ring structure occupies an antiperiplanar position more easily than the fivemembered ring structures. We believe that such thermal stabilities of the polymers would very effectively prevent the resist pattern from melting during high temperature manufacturing processes.

\subsection{Optical density of the resists}

We examined the optical densities of the formulated resist films containing $2 \mathrm{wt} \%$ of TPSOTf on quartz substrates. (Table 5) Since each resist shows acceptable transparency, the protective groups in polymers do not affect photoabsorption. This indicates that these resists have a moderate optical density for $193-\mathrm{nm}$ patterning, which is similar to MLMA-MAdMA resist.

\subsection{Lithographic performance}

Resist compositions and baking conditions are listed in Table 6. EL (Ethyl lactate) was not an acceptable solvent for the GBL resist because it needed four different solvents to form uniform films by spincoating. Based on this observation, we chose PGMEA for a resist solvent in the others. The baking conditions used in this investigation were not optimized for 193-nm exposure. We settled on the conditions according to the results of deep-UV $(\lambda=254$ $\mathrm{nm}$ ) contact exposure.

We investigated the line and space $(\mathrm{L} / \mathrm{S})$ pattern profiles after exposure using an ArF excimer laser exposure system followed by PEBs and development in a $2.38 \%$ TMAH solution. The drastic effect of protective groups on the lithographic performance was observed in the series of resists. Figure 2 shows our first experiment in GBL, HGB, 3-THF, MBL and $\mathrm{PC}$ resist. The lithographic results agree to some extent with our results on the acidic deprotection of the protective groups. The protective groups with very weak or no deprotection capabilities (GBL, 3THF and PC) exhibited poor patterning results. Although HGB and MBL displayed relatively weak

Table 6. Resist Compositions and Baking Conditions

\begin{tabular}{ccc}
\hline resist & solvent & Prebake $/$ PEB $\left(60 \mathrm{~s} /{ }^{\circ} \mathrm{C}\right)$ \\
\hline GBL & EL, GBL, & $120 / 100$ \\
& xylene, CH* \\
HGB & PGMEA, GBL & $135 / 110$ \\
3-THF & $\uparrow$ & $120 / 100$ \\
PC & $\uparrow$ & $\uparrow$ \\
MBL & $\uparrow$ & $\uparrow$ \\
* Cyclohexanone &
\end{tabular}




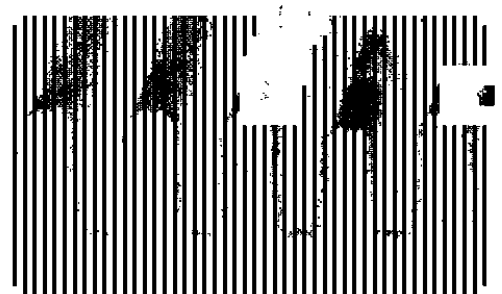

GBL $\left(0.2 \mu \mathrm{m}, 26 \mathrm{~mJ} / \mathrm{cm}^{2}\right)$

$200 \mathrm{~nm}$

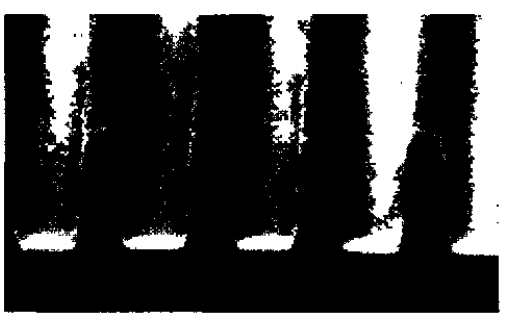

$\operatorname{MBL}\left(0.2 \mu \mathrm{m}, 26 \mathrm{~mJ} / \mathrm{cm}^{2}\right)$

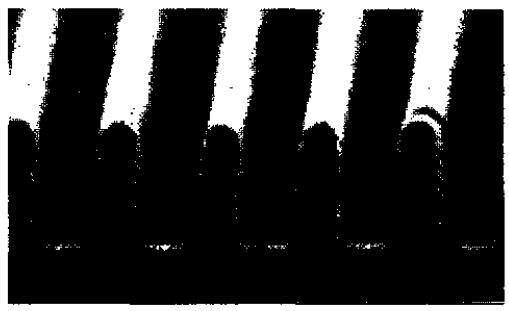

HGB $\left(0.2 \mu \mathrm{m}, 13.2 \mathrm{~mJ} / \mathrm{cm}^{2}\right)$

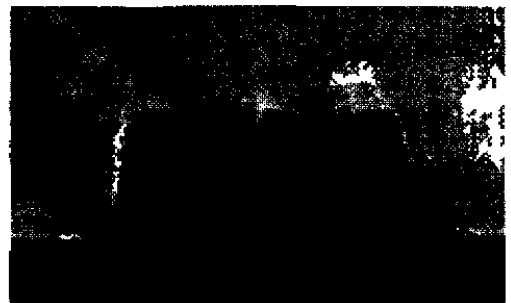

$\mathrm{PC}\left(0.24 \mu \mathrm{m}, 23 \mathrm{~mJ} / \mathrm{cm}^{2}\right)$

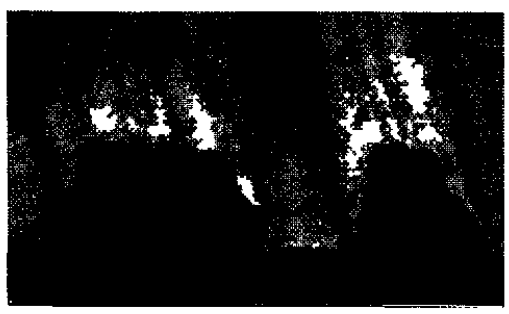

3-THF (0.2 $\left.\mu \mathrm{m}, 17 \mathrm{~mJ} / \mathrm{cm}^{2}\right)$

Figure 2. SEM micrographs depicting positive images of 0.2 - and $0.24-\mu \mathrm{m} \mathrm{L} / \mathrm{S}$ patterns in $0.5-\mu \mathrm{m}$-thick films of GBL, HGB, 3-THF, MBL and PC resists. The images exposed at $193 \mathrm{~nm}(\mathrm{NA}=0.55)$.

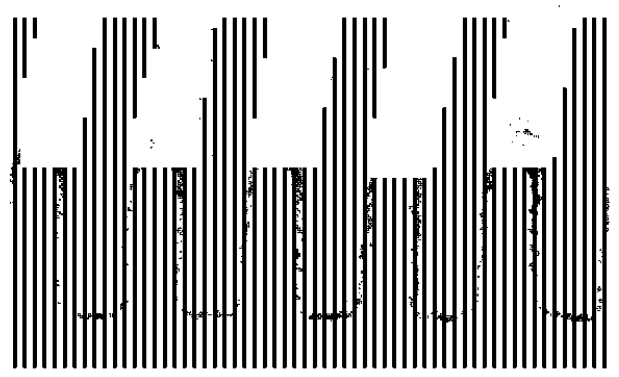

Figure 3. SEM micrographs of $0.185 \mu \mathrm{m} \mathrm{L} / \mathrm{S}$ patterns in HGB (left, $13.2 \mathrm{~mJ} / \mathrm{cm}^{2}$ ) and MBL (right, $26 \mathrm{~mJ}$ / $\mathrm{cm}^{2}$ ) resists after 193-nm exposure. The thickness of the films were $0.5 \mu \mathrm{m}$.

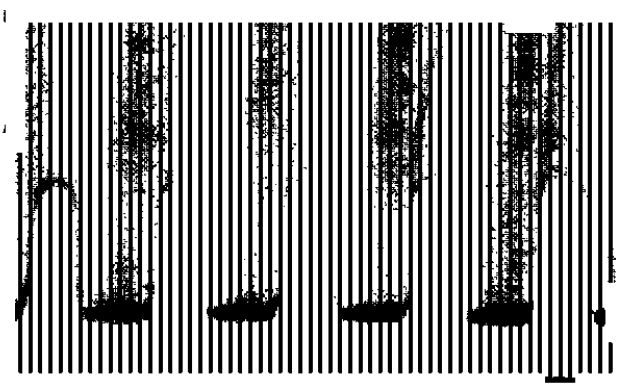

$200 \mathrm{~nm}$ deprotection capabilities in our experiments, they exhibited good lithographic results. We attribute this phenomena to the difference of the acidity and the reaction conditions between $\mathrm{DCl} / \mathrm{D}_{2} \mathrm{O}$ in $\mathrm{DMSO}-d_{6}$ and triflic acid in resist films.

In terms of pattern profiles, we observed that the pattern changed substantially when using the tetrahydrofuranyl group. The pattern appeared shrank at the surface of the 3-THF resist. We think that this phenomenon was caused by the shrinkage during the rinsing process of the swelled resist in the alkali developer. Undercuts at the resist bottoms were also observed in this resist. All lactone protected resists (GBL, HGB and MBL) displayed round-shaped patterns at their resist top, suggesting that the alkali solubility of the adjacent exposed areas was somewhat high. This means that the lactones are highly polar (hydrophilic). The GBL resist gave incomplete patterns at $0.2-\mu \mathrm{m}$ dimensions. This observation implies the alkali solubility of the exposed region was not so high as to resolve the patterns completely due to the lack of the acid cleavability of the lactone.

We obtained relatively sloped patterns without standing waves in the MBL resist. We think that the acid diffusion in this resist was somewhat large. The HGB resist gave satisfactory profiles with standing waves in the resist patterns. Although the structures of the protective groups were very similar (with or without methyl group), the difference in the resist profiles between MBL and HGB was noteworthy. We attribute the difference to the reactivity of the lactones to triflic acid in resist films. The standing waves in HGB explain that the acid diffusion in the exposed area was smaller than in MBL.

In the PC resist, there were much residue over the resist patterns. The alkali solubility of the exposed region seemed to be very low because the cyclic carbonate was inert to the condition. Since the formulated resist patterns peeled off severely, the matrix resin of the resist could be very hard and it made 
the film very brittle. We could not obtain less than $0.24-\mu \mathrm{m}$ patterns in this resist.

Among these resists, the minimum features which we obtained were $0.185 \mu \mathrm{m}$ in the HGB and the MBL resists. (Figure 3) The conditions were the same in Figure 2. We obtained relatively thin and sloped patterns in the MBL resist. On the other hand, approximately equal widths were obtained in L/S patterns in the HGB resist. The results are very surprising because the deprotection capabilities of the lactones in HGB and MBL were much lower than that of MLMA in our experiments. We think that their deprotection capabilities in the resist films is much higher than in DCl/DMSO- $d_{6}$.

Through this investigation, we found that polarity and acid cleavability of the protective group were necessary to obtain good lithographic performance. Based on the profiles and the resolution perspective, the acid cleavable lactones are favorable for use as the protective group in combination with 2-methyl2-adamantyl group in a methacrylate polymer. Most of them, 3-hydroxy- $\gamma$-butyrolactone (HGB) is the best protective group in this experiment. Furthermore, the material is commercially available and the cost is lower than that of mevalonic lactone. We think that 3-hydroxy- $\gamma$-butyrolactone is a suitable protective group in our 193-nm resist for mass production.

\section{Summary}

New polymers based on polar protective group protected methacrylates and 2-methyl-2-adamantyl methacrylate copolymers have been prepared. We have found that polar and acid cleavable protective groups in the alicyclic methacrylate resist exert a remarkable influence on the resist profiles and resolution.

In combination with the adamantyl group in a methacrylate resist, a polar protective group without acid cleavability gave more than $0.2-\mu \mathrm{m}$ patterns, but a polar and acid cleavable protective groups easily afforded less than $0.2-\mu \mathrm{m}$ patterns after $2.38 \%$ TMAH development. In particular, the combination of 3-hydroxy- $\gamma$-butyrolactone and 2-methyl-2-adamantanol for protective groups in a methacrylate resist provided acceptable lithographic performance. Using the commercially available 3-hydroxy- $\gamma$-butyro-lactone, the material could be prepared at a lower cost and large quantity.

Further chemical and lithographic evaluations are currently in progress.

\section{Acknowledgments}

We thank A. Kotachi and S. Takechi (Fujitsu Ltd.) for assistance with the ArF evaluations. We also thank I. Hanyu (Fujitsu Ltd.) for useful discussions.

\section{References}

1. K. Nozaki, K. Watanabe, T. Namiki, M. Igarashi, Y. Kuramitsu, and E. Yano, Symposium on Polymers for Microelectronics and Photonics at Pacifichem'95 (1995) No.321.

2. K. Nozaki, K. Watanabe, and E. Yano, Jpn. J. Appl. Phys., 35, Pt. 2, L 528-530 (1996).

3. K. Nozaki, K. Watanabe, E. Yano, A. Kotachi, S. Takechi, and I. Hanyu, J. Photopolym. Sci. Technol., 9 (3), 509 (1996).

4. S. Takechi, M. Takahashi, A. Kotachi, K. Nozaki, E. Yano, and I. Hanyu, J. Photopolym. Sci. Technol., 9(3), 475 (1996).

5. S. Takechi, Y. Kaimoto, K. Nozaki, and N. Abe, J. Photopolym. Sci. Technol., 5 (3), 439 (1992).

6. Y. Kaimoto, K. Nozaki, S. Takechi, and N. Abe, Proc. SPIE, 1672, 66 (1992).

7. K. Nozaki, Y. Kaimoto, M. Takahashi, S. Takechi, and N. Abe, Extended Abstracts, 53rd Autumn Meeting JSAP, 504 (1992).

8. K. Nozaki, Y. Kaimoto, M. Takahashi, S. Takechi, and N. Abe, Chem. Mater., 6 (9), 1492 (1994).

9. S. Takechi, M. Takahashi, and I. Hanyu, Proc. OCG Microlithography, INTERFACE'95, 43 (1996).

10. M. Endo, K. Hashimoto, K. Yamashita, T. Matsuo, Y. Tani, M. Sasago, and N. Nomura, IEDM Tech. Digest, 45 (1992).

11. R. D. Allen, G. M. Wallraf, R. A. Dipietro, D. C. Hofer, and R. R. Kunz, J. Photopolym. Sci. Technol., 8 (4), 623 (1995); R. D. Allen, R. Sooriyakumaran, J. Optiz, G. M. Wallraff, G. Breyta, R. A. Dipietro, D. C. Hofer, R. R. Kunz, U. Okoroanyanwu, and C. G. Willson, J. Photopolym. Sci. Technol., 9 (3), 465 (1995)

12. K. Nakano, K. Maeda, S. Iwasa, J. Yano, Y. Ogura, and E. Hasegawa, Proc. SPIE, 2195,194 (1994); K. Maeda, K. Nakano, T. Ohfuji, and E.Hasegawa, Proc. SPIE, 2724, 377 (1996)

13. N. Shida, T. Ushirogouchi, K. Asakawa, and M. Nakase, J. Photopolym. Sci. Technol., 9 (3), 457 (1996).

14. R. D. Allen, R. Sooriyakumaran, J. Optiz, G. M. Wallraff, R. A. Dipietro, G. Breyta, D. C. Hofer, R. R. Kunz, S. Jayaraman, R. Shick, B.Goodall, U. Okoroanyanwu, and C. G. Willson, Proc. SPIE, 2724, 334 (1996).

15. T. I. Wallow, F. M. Houlihan, O. Nalamasu, E. A. Chandross, T. X. Neenan, E. Reichmanis, Proc. SPIE, 2724, 355 (1996)

16. S. Choi, Y. Kang, D. Jung, C. Park, and Y. Koh, SPIE Abstract, No. 3049-07, 62 (1997) 